\title{
Report of working group of the British Association of Perinatal Medicine and Neonatal Nurses Association on categories of babies requiring neonatal care
}

There should be four levels of care: level 1 intensive care (maximal intensive care), level 2 intensive care (high dependency intensive care), special care, and normal care.

\section{Definitions of neonatal care}

LEVEL 1 INTENSIVE CARE (MAXIMAL INTENSIVE CARE)

Care given in an intensive care nursery which provides continuous skill supervision by qualified and specially trained nursing and medical staff. Such care includes support of the infant's parents.

\section{LEVEL 2 INTENSIVE CARE (HIGH DEPENDENCY INTENSIVE CARE) \\ Care given in an intensive or special care nursery which provides continuous skilled supervision by qualified and specially trained nursing staff who may care for more babies than in level 1 intensive care. Medical supervi- sion is not so immediate as in level 1 intensive care. Care includes support of the infant's parents.}

\section{SPECIAL CARE}

Care given in a special care nursery, transitional care ward, or postnatal ward which provides care and treatment exceeding normal routine care. Some aspects of special care can be undertaken by a mother supervised by qualified nursing staff. Special nursing care includes support and education of the infant's parents.

NORMAL CARE

Care given by the mother or mother substitute with medical or neonatal nursing advice if needed.

\section{Clinical categories of neonatal care}

LEVEL 1 INTENSIVE CARE (MAXIMAL INTENSIVE CARE)

Level 1 intensive care should be provided for these babies:

(1) Receiving assisted ventilation (including intermittent positive airway pressure, intermittent mandatory ventilation, and constant positive airway pressure) and in the first 24 hours after its withdrawal.

(2) Of less than 27 weeks' gestation for the first 48 hours after birth.

(3) With birth weight of less than $1000 \mathrm{~g}$ for the first $\mathbf{4 8}$ hours after birth.
(4) Who require major emergency surgery for the preoperative period and post operatively for $\mathbf{4 8}$ hours.

(5) On the day of death.

(6) Being transported by a team including medical and nursing staff.

(7) Who are receiving peritoneal dialysis.

(8) Who require exchange transfusions complicated by other disease processes.

(9) With severe respiratory disease in the first 48 hours of life requiring a fractional inspiratory oxygen $\left(\mathrm{Fio}_{2}\right)$ of $>0 \cdot 6$.

(10) With recurrent apnoea needing frequent intervention, for example over five stimulations in eight hours or resuscitation with intermittent positive pressure ventilation (IPPV) two or more times in 24 hours.

(11) With significant requirements for circulatory support, for example inotropes, three or more infusions of colloid in 24 hours, or infusions of prostaglandins.

LEVEL 2 INTENSIVE CARE (HIGH DEPENDENCY INTENSIVE CARE)

Level 2 intensive care should be provided for these babies:

(1) Requiring total parenteral nutrition.

(2) Who are having convulsions.

(3) Being transported by a trained skilled neonatal nurse alone.

(4) With arterial line or chest drain.

(5) With respiratory disease in the first 48 hours of life requiring an $\mathrm{Fio}_{2}$ of 0.4-0.6.

(6) With recurrent apnoea requiring stimulation up to five times in an eight hour period or any resuscitation with IPPV.

(7) Who require an exchange transfusion alone.

(8) Who are more than 48 hours' postoperative and require complex nursing procedures.

(9) With tracheostomy for first two weeks.

SPECIAL CARE

Special care should be provided for these babies:

(1) Requiring continuous monitoring of respiration or heart rate or by transcutaneous transducers.

(2) Receiving additional oxygen.

(3) With tracheostomy after first two weeks.

(4) Being given intravenous glucose and electrolyte solutions.

(5) Who are being tube fed.

(6) Who have had minor surgery in the previous 24 hours. 
(7) Who require terminal care but not on the day of death.

(8) Being barrier nursed.

(9) Undergoing phototherapy.

(10) Receiving special monitoring (for example frequent glucose or bilirubin estimations).

(11) Needing constant supervision (for example babies whose mothers are drug abusers).

(12) Being treated with antibiotics.

\section{Resources required for neonatal care STAFFING}

The British Paediatric Association (BPA) recommendation and the Neonatal Nurses Association guidelines for staffing are as follows but are subject to local conditions and are not, as yet, founded on research on dependency levels. On presentation of appropriate research findings these may require amending.

\section{Nursing staff}

Trained nurses hold registered general nurse, registered midwife, registered sick children's nurse, or the enrolled nurse certificates. Qualified nurses hold a certificate in intensive care of newborn, for example, English National Board course 405, 409, 904, 904A certificate or Joint Board of Clinical Studies certificate, that is $400,401,402$. Maximal intensive care (level 1 intensive care) requires 5.5 nurses whole time equivalent (wte), qualified and trained per cot, high dependency intensive care (level 2 intensive care) requires 3.5 nurses (wte) qualified and trained per cot, and special care requires 1.0 nurses (wte) qualified per cot. Neonatal transport: additional provision of staff will be required by centres offering to transport sick infants.

\section{Medical staff}

Maximal intensive care (level 1 intensive care)-minimum medical staffing should consist of both an experienced paediatric registrar and senior house officer on duty and available in the intensive care area at all times with an appropriately trained consultant in charge.

High dependency intensive care (level 2 intensive care)-an experienced senior house officer on duty and available in the high dependency area at all times with a more senior member of staff on call and a consultant paediatrician in charge.

Special care-minimum medical staff for 24 hour cover: an appropriately experienced senior house officer on duty, an experienced more senior member of staff on call, and a consultant paediatrician in charge.

Neonatal transport-additional provision of staff will be required by centres offering to transport sick infants.

\section{EQUIPMENT}

The BPA recommends the following equipment and facilities:
Maximal intensive care (level 1 intensive care)

The following equipment should be available for each baby:

- Intensive care incubator, or unit with overhead hearing (1)

- Respiratory or apnoea monitor (1)

- Heart rate monitor (1)

- Intravascular blood pressure transducer or surface blood pressure recorder (1)

- Transcutaneous oxygen tension monitor, or intravascular oxygen transducer, or saturation oxygen tension monitor (1)

- Transcutaneous carbon dioxide tension monitor (1)

- Syringe pumps (2)

- Infusion pumps (2)

- Ventilator (1)

- Continuous temperature monitor (1)

- Phototherapy unit (1)

- Ambient oxygen monitor (1)

- Facilities for frequent blood gas analysis using micromethods

- Facilities for frequent biochemical analysis including glucose, bilirubin, and electrolytes by micromethods

- Facilities for necropsy by an appropriately trained paediatric pathologist

- Access to equipment for visualisation of organs such as the brain

- Access to equipment for radiological examination

- Access to transport incubator with transport ventilator

High dependency intensive care (level 2 intensive care)

Same as level 1 intensive care but without a ventilator.

Special care

The following equipment and facilities should be available for each baby who may need it:

- Incubator or cot adequate for temperature control (1)

- Ambient oxygen analyser (1)

- Apnoea alarm (1)

- Heart rate monitor (1)

- Infusion pump (1)

- Phototherapy unit (1)

- Ventilator to be used for short term ventilation (1)

- Access to frequent blood gas analysis using micromethods

- Access to biochemical analysis (including glucose, bilirubin, and electrolytes) by micromethods

- Facilities for necropsy by an appropriately trained paediatric pathologist

- Access to equipment for radiological examination

- Access to transport incubator with transport ventilator

Special care may take place on a postnatal ward, particularly in an area specially set aside for the purpose.

Units providing level 2 intensive care or special care only should have the availability of equipment for maintaining babies in optimal condition before transfer. 